\title{
Definitive observation of the dark triplet ground state of charged excitons in high magnetic fields
}

\author{
G. V. Astakhov ${ }^{1,2}$, D. R. Yakovlev ${ }^{2,3}$, V. V. Rudenkov ${ }^{4}$, P. C. M. Christianen ${ }^{4}$, T. Barrick ${ }^{5}$, \\ S. A. Crooker $^{5}$, A. B. Dzyubenko ${ }^{6}$, W. Ossau ${ }^{1}$, J. C. Maan $^{4}$, G. Karczewski ${ }^{7}$, and T. Wojtowicz ${ }^{7}$ \\ ${ }^{1}$ Physikalisches Institut der Universität Würzburg, 97074 Würzburg, Germany \\ ${ }^{2}$ A.F.Ioffe Physico-Technical Institute, Russian Academy of Sciences, 194021, St.Petersburg, Russia \\ ${ }^{3}$ Experimentelle Physik 2, Universität Dortmund, 44221 Dortmund, Germany \\ ${ }^{4}$ High Field Magnet Laboratory, University of Nijmegen, 6525 ED Nijmegen, The Netherlands \\ ${ }^{5}$ National High Magnetic Field Laboratory, Los Alamos, New Mexico 87545, USA \\ ${ }^{6}$ Department of Physics, California State University at Bakersfield, Bakersfield, CA 93311, USA \\ ${ }^{7}$ Institute of Physics, Polish Academy of Sciences, PL-02668 Warsaw, Poland
}

(Dated: November 14, 2018)

\begin{abstract}
The ground state of negatively charged excitons (trions) in high magnetic fields is shown to be a dark triplet state, confirming long-standing theoretical predictions. Photoluminescence (PL), reflection, and PL excitation spectroscopy of CdTe quantum wells reveal that the dark triplet trion has lower energy than the singlet trion above 24 Tesla. The singlet-triplet crossover is "hidden" (i.e., the spectral lines themselves do not cross due to different Zeeman energies), but is confirmed by temperature-dependent PL above and below $24 \mathrm{~T}$. The data also show two bright triplet states.

PACS numbers: 71.35.Pq, 71.35.Ji, 78.66.Hf
\end{abstract}

A central problem found in atomic, solid state, and nuclear physics is the case of a three-particle system of fermions, bound together by long-range Coulomb interactions. In atomic physics, this situation is most simply realized by the two-electron hydrogen ion, $\mathrm{H}^{-}$, in which the two identical electrons can exist in either a singlet or triplet state with total electron spin $S_{e}=0$ or 1, depending on external parameters. The semiconductor analog of the $H^{-}$ion is the negatively charged exciton (trion), consisting of two conduction electrons bound to a single valence hole. Optical signatures from trions have been observed in GaAs, CdTe, and ZnSe quantum wells (QWs) 1, 2, 3, 4]. Unlike the $H^{-}$ion, the hole and two electrons comprising the trion have comparable masses and typically experience strong QW confinement in one dimension, making trions a genuine quantum three-particle system with Coulomb interactions for which no general analytical solutions exist.

Much attention has focused on the evolution of trion optical signatures with applied magnetic field 5, 6, 7. 8, 9]. In the limit of zero magnetic field, theory predicts just one bound trion state: the $S_{e}=0$ singlet trion $\left(T_{s}\right)$ 10, 11]. This is consistent with Hill's theorem 12], which states that the $\mathrm{H}^{-}$ion (with an infinitely massive proton) supports exactly one bound singlet state. In the opposite limit of extremely high magnetic fields, it can be rigorously shown that a $S_{e}=1$ triplet is the only bound trion state in a strictly 2D system 11, 13. Modelindependent symmetry considerations 14] demonstrate that this lowest triplet state is "dark" $\left(T_{t d}\right)$ (i.e., optically inactive), due to the exact selection rules imposed by spatial axial and translational symmetries that exist in a disorder-free QW. Thus, at finite magnetic fields one expects both singlet and triplet bound trions 11, 13. More importantly, at some critical magnetic field $B_{c}$ the spin configuration of the trion ground state must cross over from the singlet to the triplet. Theoretical estimates suggest this crossover field is very large $\left(B_{c}>20 \mathrm{~T}\right)$ and depends sensitively on the strength of the Coulomb interaction (dielectric constant) and the details of the QW confinement 11, 15, 16, 17, 18]. Numerical calculations also point to the existence of weakly-bound, optically active "bright" triplet states $\left(T_{t b}\right)$, although there is large disparity amongst the predicted regions of stability and binding energies [15, 16]. Distinction between $T_{t d}$ and $T_{t b}$ is due to orbital motion and is not related to the spin selection rules [14, 15, 16]. Note, $T_{t d}$ and $T_{t b}$ have identical spin configuration.

In this Letter we present conclusive evidence that the high-field $\left(B>B_{c}=24 \mathrm{~T}\right)$ ground state of negativelycharged trions in CdTe-based QWs is 1) a triplet state, and 2) optically dark, - i.e., it has no absorption oscillator strength. Three distinct and complementary polarization-resolved spectroscopies - photoluminescence (PL), reflection, and PL excitation (PLE) 19] - proved to be essential for identifying and conclusively determining the spin properties of trions in magnetic fields below and above the singlet-triplet crossover field $B_{c}$. As such, this work represents what is to our knowledge the first comprehensive picture of the evolution of the trion ground state's spin over a complete range of magnetic fields. Two important aspects of the singlet-triplet crossover, revealed particularly in high-field PL spectra, require careful accounting of the Zeeman energies of the initial trion and the final electron states. First, the actual crossover point is shifted to much lower fields $\left(B_{c}=24 \mathrm{~T}\right)$ than the $\sim 70 \mathrm{~T}$ that is expected when Zeeman energies are disregarded. Second, and less obvious, the singlet-triplet crossing is hidden from direct observation - i.e., the measured $T_{s}$ and $T_{t d}$ PL peaks themselves do not cross. This is because, following emission, the spin (and therefore energy) of the final remaining electron is different for $T_{s}$ 

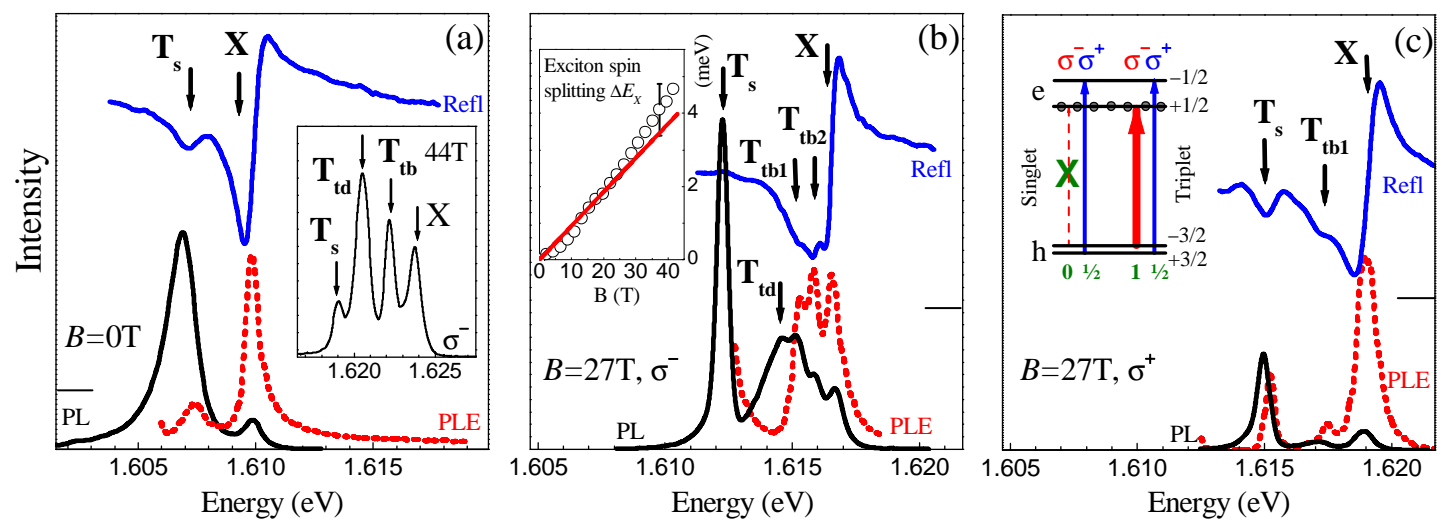

FIG. 1: PL, PLE, and reflectivity (Refl) spectra of a $120 \AA \mathrm{CdTe} / \mathrm{Cd}_{0.85} \mathrm{Mg}_{0.15} \mathrm{Te} \mathrm{QW}$ with $n_{e}=2 \times 10^{10} \mathrm{~cm}^{-2}$ at $T=1.3 \mathrm{~K}$. The singlet $\left(T_{s}\right)$, dark triplet $\left(T_{t d}\right)$, two bright triplet $\left(T_{t b 1}\right.$ and $\left.T_{t b 2}\right)$ trions, and the neutral exciton $(X)$ are clearly seen. (a) Spectra at $0 \mathrm{~T}$. Inset: $\sigma^{-} \mathrm{PL}$ at $B=44 \mathrm{~T}$. (b,c) Spectra at $27 \mathrm{~T}$ in $\sigma^{-}$and $\sigma^{+}$polarizations. Inset (b): $X$ Zeeman splitting (circles) and electron splitting (line) for $g_{e}=-1.60$. Inset (c): Schematic of transitions leading to photocreation of $T_{s}$ and $T_{t b}$. Transition probabilities are coded by the arrow thickness, dashed line means the forbidden transition.

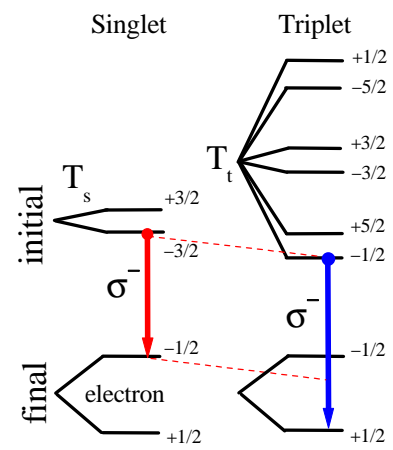

FIG. 2: Schematic of the hidden singlet-triplet crossover of trion states in CdTe QWs. At high magnetic fields, the lowest dark triplet state has less energy than the lowest singlet. After recombination, however, the remaining electron is left in different spin states. Thus, the observed triplet PL energy remains larger than that of the singlet, as indicated by the lengths of the arrows.

and $T_{t d}$. Rather, the crossover is revealed by an exchange of intensity between the $T_{s}$ and $T_{t d}$ lines in PL, and by their temperature dependence above and below $B_{c}$.

Single $120 \AA \mathrm{CdTe} / \mathrm{Cd}_{0.85} \mathrm{Mg}_{0.15} \mathrm{Te} \mathrm{QWs}$ were grown by molecular beam epitaxy on (100)-oriented GaAs substrates by wedge-doping, which allows different electron densities $n_{e}$ on the same wafer [20]. The data presented here were from a structure with $n_{e}=2 \times 10^{10} \mathrm{~cm}^{-2}$. Polarized optical spectra were measured at low temperatures $(1.3-10 \mathrm{~K})$ and in magnetic fields applied parallel to the growth axis (Faraday geometry). DC fields to $33 \mathrm{~T}$ (Nijmegen) and pulsed fields to $44 \mathrm{~T}$ (Los Alamos) were used. Ti:Sapphire or He-Ne lasers with power density $<1 \mathrm{~W} / \mathrm{cm}^{2}$ were used for excitation via fibers or by direct optical access. Circularly polarized light was used to resolve the exciton and trion spin orientation.

Zero-field optical spectra are shown in Fig. 1h, where the well-known pair of resonances associated with the neutral exciton $X$ and singlet trion $T_{s}$ are clearly seen in PL, PLE, and reflectivity. Triplet states, being unbound at zero field, are not observed. Linewidths are $<1.5 \mathrm{meV}$, much smaller than the $3 \mathrm{meV}$ trion binding energy (taken as the energy difference between $X$ and $T_{s}$ lines). The $T_{s}: X$ ratio of oscillator strengths is 1:9 (from PLE and reflectivity), permitting evaluation of the 2DEG density 21]. At 44 Tesla (inset), the PL spectra develop two additional strong peaks between the $X$ and $T_{s}$ lines, which we assign to dark and bright triplets based on their polarization, energy, and evolution with magnetic fields as discussed below.

In finite magnetic fields, correct assignment of the various optical transitions to the proper exciton or trion state is essential. Figures 1(b,c) show the polarized optical spectra at $27 \mathrm{~T}$. The neutral exciton $X$ is readily identified in reflectivity, where it dominates all other resonances, exhibits equal oscillator strength in both $\sigma^{+}$and $\sigma^{-}$polarizations, and also appears in an undoped reference sample. The features observed at the same energy in PL and PLE spectra are therefore also assigned to $X$. Note that while $X$ is strong in PLE spectra, it is weak in PL due to thermalization to lower-lying trion states.

At $27 \mathrm{~T}$, the $X$ Zeeman splitting is $\sim 2.7 \mathrm{meV}$. The field dependence of the $X$ and electron Zeeman splittings are shown in the inset. The latter, determined by spin-flip Raman scattering [22], indicates an electron gfactor $g_{e}=-1.60$. The exciton spin splitting, $\Delta E_{X}=$ $\left(g_{h h}-g_{e}\right) \mu_{B} B$, therefore implies a small heavy-hole gfactor $\left(\left|g_{h h}\right|<0.2\right)$ which actually changes sign at $\sim 18 \mathrm{~T}$.

Trion formation involves a photocreated electron-hole pair and a background electron from the 2DEG. In high magnetic fields, when the 2DEG is totally spin polarized ( $B>4 \mathrm{~T}$ in this sample), singlet and triplet trion states can be identified by their distinct polarizations in PLE, reflectivity, and PL spectra. The singlet trion $T_{s}$ with the lowest Zeeman energy has net spin projection 


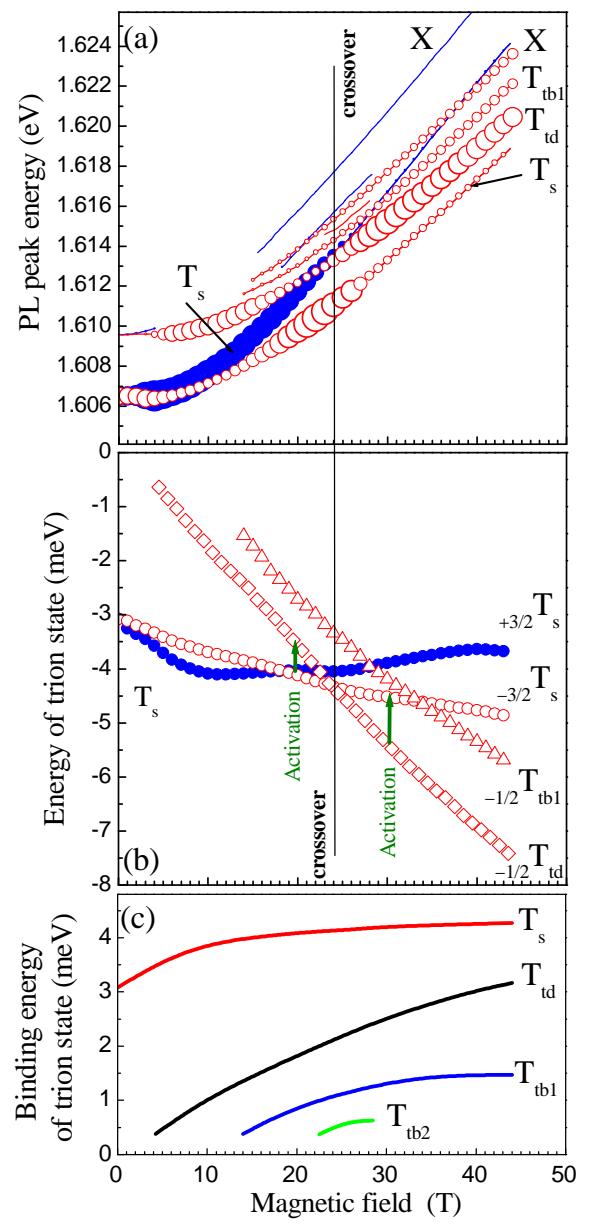

FIG. 3: (a) Evolution of trion PL peaks in $\sigma^{-}$(open) and $\sigma^{+}$(solid) polarizations. Symbol size indicates PL intensity. (b) Energies of the $T_{s}$ (circles), $T_{t d}$ (diamonds), and $T_{t b 1}$ (triangles) trion states, measured from the neutral exciton $X$, showing $B_{c}=24 \mathrm{~T}$. Arrows mark the thermal activation processes measured in Fig. 4 (c) Trion binding energies from Coulomb interactions alone (no Zeeman).

$S_{z}=S_{e z}+S_{h z}=0-\frac{3}{2}=-\frac{3}{2}$ (see Fig. 2). This singlet emits a $\sigma^{-}$photon upon recombination, leaving the remaining electron in the upper $\left(-\frac{1}{2}\right)$ Zeeman state. Formation of $T_{s}$, requiring a photoexcited electron with spin antiparallel to the 2DEG electrons, should therefore exhibit a strong PLE and reflectivity resonance only in $\sigma^{+}$ polarization, as observed. Indeed, $T_{s}$ is quite strong in $\sigma^{+}$PLE and reflectivity, and completely absent in the $\sigma^{-}$reflectivity (its absence in $\sigma^{-}$PLE is obscured by scattered light, where we always detect $\sigma^{-}$emission).

In contrast, triplet trions are predominantly polarized opposite to the singlet. The lowest energy triplet has net spin $S_{z}=S_{e z}+S_{h z}=+1-\frac{3}{2}=-\frac{1}{2}$. This triplet also emits a $\sigma^{-}$photon upon recombination, but unlike $T_{s}$, leaves the remaining electron in the lower $\left(+\frac{1}{2}\right)$ Zeeman state. This distinction will prove important shortly, when discussing the hidden singlet-triplet crossover. Formation of triplet trions, requiring predominantly spin- parallel electrons, should therefore exhibit resonances largely in the $\sigma^{-}$PLE and reflectivity. Indeed, two additional resonances in $\sigma^{-}$PLE and reflectivity are clear, and both have corresponding $\sigma^{-}$PL emission (Fig. 1). We therefore assign these lines to two "bright" (optically active) triplet trion states $T_{t b 1}$ and $T_{t b 2}$. Most importantly, however, an additional strong $\sigma^{-}$polarized PL peak is seen at energy $1.6145 \mathrm{eV}$. It has no counterpart in PLE or reflectivity spectra, meaning that the corresponding transition has no oscillator strength and is optically inactive. Thus, we assign this PL peak to the dark triplet $T_{t d}$. It has the largest binding energy among the triplet states, consistent with theoretical predictions [11, 15, 16]. The reason dark triplet PL appears at all is due to the small but nonzero probability of allowed radiative recombination via disorder scattering [14] or interaction with excess electrons, as demonstrated even in low density 2DEGs $\left(n_{e} \sim 10^{10} \mathrm{~cm}^{-2}\right)$ [24].

Figure 3a shows the energy shifts of the trion PL with magnetic field, where symbol size indicates the PL intensity and weak transitions are traced by lines. We concentrate primarily on the evolution of the $T_{s}$ and $T_{t d}$ peaks. For all accessible fields $(0-44 \mathrm{~T})$, the $T_{s} \mathrm{PL}$ peak occurs at the lowest measured energy. However, at about $24 \mathrm{~T}$, the $T_{s}$ PL intensity is significantly redistributed in favor of $T_{t d}$, strongly suggesting that the bound dark triplet has crossed the singlet to become the trion ground state. However, the observed PL lines themselves do not cross. This seeming contradiction is resolved by recalling that the electrons which remain after recombination of $T_{s}$ and $T_{t d}$ reside in the upper $\left(-\frac{1}{2}\right)$ and lower $\left(+\frac{1}{2}\right)$ spin states respectively, and these final states are split by the electron Zeeman energy $\Delta E_{e}=\mu_{B}\left|g_{e}\right| B$. As shown schematically in Fig. 2, the $T_{t d}$ state can have lower energy than $T_{s}$, but emission from $T_{t d}$ may still have the greater energy. At the crossover field $B_{c}$, when the $T_{s}$ and $T_{t d}$ states themselves have identical energies, the energy of $T_{t d}$ emission still exceeds the energy of $T_{s}$ emission by exactly $\Delta E_{e}$. We describe the change of trion ground state as a "hidden" crossing between $T_{s}$ and $T_{t d}$ [23].

The hidden crossover is revealed particularly well by the temperature dependence of the trion PL peaks above and below $B_{c}=24 \mathrm{~T}$ (Fig. 4). At $20 \mathrm{~T}$ (below $B_{c}$ ), increasing the temperature from 1.3 to $5.9 \mathrm{~K}$ depopulates the $T_{s}$ state in favor of $T_{t d}$, implying thermal excitation of trions from a singlet ground state to a higher-energy dark triplet. In contrast, the same temperature increase at $30 \mathrm{~T}$ (above $B_{c}$ ) has the opposite effect - an increase in $T_{s}$ emission and a reduction in $T_{t d}$ emission, implying thermal excitation from a dark triplet ground state to a higher-lying singlet state. In other words, the trion ground state has crossed over from singlet to dark triplet. A fit to the ratio of PL intensities vs. temperature (the inset of Fig. (4) reveals that the radiative recombination times of the trion states satisfy $t_{t d} \gg t_{s}$ 25], independently confirming the identification of $T_{t d}$ as a dark state.

Whereas the Zeeman splitting of the final electron states causes the "hidden" nature of the crossover, the 


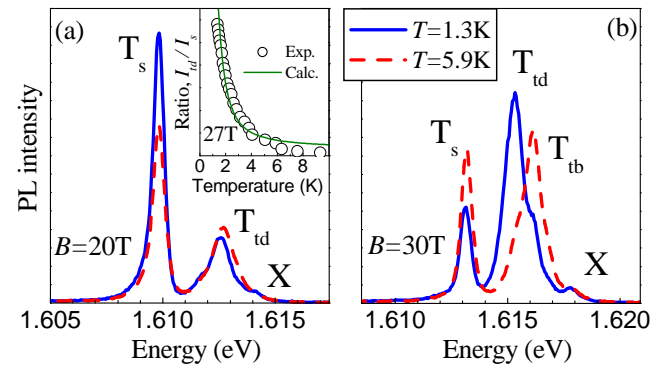

FIG. 4: The temperature dependence of PL spectra measured in $\sigma^{-}$polarization at $20 \mathrm{~T}$ (a) and $30 \mathrm{~T}(\mathrm{~b})$. Inset: the $T_{t d}: T_{s}$ intensity ratio vs. temperature. The line is a calculation based on a two-level model with $0.45 \mathrm{meV}$ energy splitting.

different Zeeman splittings of the initial $T_{s}$ and $T_{t d}$ states has an additional important consequence. Namely, the crossover occurs at a much lower magnetic field than it would in the absence of Zeeman effects. Figure 3b shows the initial energies of all trion states, measured with respect to the "center-of-gravity" of the neutral exciton Zeeman doublet, which accounts for the overall diamagnetic shift. Each trace has a contribution from the trion's Coulomb binding energy, as well as the additional Zeeman energy of the initial trion state. The striking feature of Fig. B is the evident crossover between $T_{t d}$ (with spin projection $S_{z}=-\frac{1}{2}$ ) and $T_{s}$ (with $S_{z}=-\frac{3}{2}$ ) which occurs at $24 \mathrm{~T}$. This value coincides very well with the field at which the PL intensity redistribution occurs. Note also that a bright triplet state $T_{t b 1}$ (with $S_{z}=-\frac{1}{2}$ ) crosses the singlet at $\sim 34 \mathrm{~T}$. For future comparison with theory, we also plot in Fig. 3: the trion binding energies resulting from Coulomb interactions alone (i.e., without Zeeman terms). It is evident that the actual crossover field is indeed reduced due to the electron Zeeman splitting $\Delta E_{e}$, without which $B_{c}$ would estimated to $\sim 70 \mathrm{~T}$, in good qualitative agreement with theoretical predictions for II-VI QWs [17].

As discussed briefly above, the data also reveal another novel feature: a second bright triplet state $T_{t b 2}$. It is detected in PL, PLE, and reflectivity spectra between $22 \mathrm{~T}$ and $28 \mathrm{~T}$ (see Figs. 1b and 3r). No experimental observations of this state have been reported to date. While CdTe QWs are characterized by strong Coulomb interactions, this enhancement is not enough to ensure binding of additional trion states because the neutral exciton relative to which a trion may or may not be bound - is also more tightly bound. Some new physics is needed here. One possibility is that trion binding energies are enhanced in these QWs by "bipolaron" effects, wherein the polarization clouds of two electrons in the trion partly overlap, lowering the total energy relative to the neutral exciton $X$ [26].

In conclusion, combined PL, PLE, and reflectivity studies reveal the detailed energy spectrum of charged trions over a wide range of magnetic fields. These trions exemplify a canonical problem of interest in many solid state, atomic, and nuclear physics problems: a three-particle spin system with long-range Coulomb interactions. We have confirmed a high-field crossover from the singlet to dark triplet trion state, upholding long-standing theoretical predictions. It has been shown that the Zeeman spin splitting of electrons both reduces the crossover field to experimentally accessible values $B_{c}=24 \mathrm{~T}$, and also causes the crossover to be "hidden" from direct observation of the emission energies themselves. We have also observed a novel feature in the spectra, an additional bound bright triplet trion state, and indicated the physics that might explain its stability.

This work was supported by the the Deutche Forschungsgemeinschaft (SFB 410), the DARPA Spins program, the NSF grant DMR-0203560, and the EU IHPARI program HPRI-CT-1999-00036.
[1] K. Kheng et al., Phys. Rev. Lett. 71, 1752 (1993).

[2] G. Finkelstein et al., Phys. Rev. Lett. 74, 976 (1995).

[3] A.J. Shields et al., Phys. Rev. B 52, 5523(R) (1995).

[4] G.V. Astakhov et al., Phys. Rev. B 60, 8485(R) (1999).

[5] G. Yusa et al., Phys. Rev. Lett. 87, 216402 (2001).

[6] T. Vanhoucke et al., Phys. Rev. B 65, 233305 (2002).

[7] C. Schüller et al., Phys. Rev. B 65, 081301(R) (2002).

[8] H. A. Nickel et al. Phys. Rev. Lett. 88, 056801 (2002).

[9] B.M. Ashkinadze et al., Phys. Rev. B 69, 115303 (2004).

[10] B. Stebe et al., Superlatt. Microstruct. 5, 545 (1989).

[11] D.M. Whittaker et. al., Phys. Rev. B 56, 15185 (1997).

[12] R. N. Hill, Phys. Rev. Lett. 38, 643 (1977).

[13] J.J. Palacios et. al., Phys. Rev. B 54, 2296(R) (1996).

[14] A.B. Dzyubenko et al., Phys. Rev. Lett. 84, 4429 (2000); Physica E 6, 226 (2000).

[15] A. Wojs et al., Phys. Rev. B 62, 4630 (2000); Physica E 8, 254 (2000).

[16] C. Riva et al., Phys. Rev. B 63, 115302 (2001).
[17] D.R. Yakovlev et al., Phys. Stat. Sol. (b) 227, 353 (2001).

[18] P. Redliński et al., Semicond. Sci. Technol. 17, 237 (2002).

[19] To our knowledge, the absorption spectra of the trion triplet was only studied in Ref. 7. However, the spectra were taken using unpolarized light leading to the loss of all the relevant information concerning the Zeeman splittings. This might strongly affect the interpretation of experimental data.

[20] T. Wojtowicz et al., Acta Physica Polonica A 94, 199 (1998).

[21] G.V. Astakhov et al., Phys. Rev. B 65, 115310 (2002).

[22] A.A. Sirenko et al., Phys. Rev. B 56, 2114 (1997).

[23] Spin structure of trions in magnetic fields (see e.g. Fig. 2) determine whether the singlet-triplet crossing is hidden or not hidden. It depends on the ratio of absolute values and signs of electron and hole g-factors, which vary with material and structure parameters. 
[24] D. Sanvitto et al., Phys. Rev. Lett. 89, 246805 (2002).

[25] The exact values used to fit the experimental data in the inset of Fig. 4 are $t_{t d}=200 t_{s}$ and $\tau=4 t_{s}$, where $\tau$ is the relaxation time between the $T_{t d}$ and $T_{s}$ states. [26] C. Riva et al., Phys. Rev. B 61, 13873 (2000). 